\title{
Replacement of Vertebral Lamina (Laminoplasty) in Surgery for Lumbar Isthmic Spondylolisthesis: 5-Year Follow-Up Results
}

\author{
Kadir Kotil \\ Department of Neurosurgery, T.C. Istanbul Arel University, School of Medicine, Istanbul, Turkey
}

Study Design: A review of clinical and radiological outcomes of lumbar laminoplasty (LL) for the treatment of isthmic spondylolisthesis (ISL).

Purpose: The single session performance of posterior lumbar interbody fusion with allograft in the anterior column and providing the realignment of the vertebrae was presented as a preliminary report earlier.

Overview of Literature: Long-term surgical outcome of cervical laminoplasty in patients has been reported. But, outcome of LL in patients is unclear.

Methods: The long-term (5 years) year follow-up results of the LL technique are reported in this retrospective study. All patients underwent preoperative and postoperative direct X-ray, computed tomography, and magnetic resonance imaging. The patients that did not respond to conservative treatment were operated. Twenty-one $(52.5 \%)$ female and $19(47.5 \%)$ male patients were included.

Results: Mean age was 43,5 years (ranges, 22-57 years). The most common symptoms were low back pain (89\%), pelvic and leg pain $(69 \%)$ and reduction in walking distance $(65 \%)$. A total of 180 pedicle screws were inserted in 40 patients; posterior lumbar interbody fusion and laminoplasty with reduction was performed in 20 patients for $L 4-L 5,12$ patients for $L 5-S 1$, 4 patients for $L 3-$ L4-L5 and 4 patients for L4-L5-S1. Ten (25\%) patients with ILL had accompanying spinal stenosis. The difference between preoperative and postoperative sagittal plane rotation and dislocation degrees and disc space heights were statistically significant in all patients $(p<0.05)$. Solid grade 4 fusion was observed in 38 patients; in only 2 patients grade 2 pseudoarthrosis developed $(5 \%)$, but these patients were asymptomatic. Visual analog scale, Prolo economical and functional scale was examined with an average followup 5.5 years.

Conclusions: LL technique has the advantages of shorter duration of operation, lack of graft donor site complications, protection of posterior column osseoligamentous structures and achievement of high fusion rates in one session.

Keywords: Posterior fusion; Lumbar; Laminoplasty; Isthmic spondylolisthesis; Long term; Follow up studies

\section{Introduction}

The technique for the surgical treatment of isthmic spondylolisthesis (ISL) is still controversial. In symptomatic patients, the outcome of surgery is better compared to conservative treatment [1]. Combined anterior and posterior intervertebral fusion may provide the best reduction, but the duration of surgery is longer and morbidity may

Received Oct 3, 2015; Revised Nov 16, 2015; Accepted Nov 16, 2015

Corresponding author: Kadir Kotil

T.C. Istanbul Arel University, Türkoba Mahallesi Erguvan Sokak No:26/K, Tepekent,

34537 Tepekent, Büyükçekmece, İstanbul, Turkey

Tel: +0216-360-12-26, Fax: +0216-302-03-91, E-mail: kadirkotil@gmail.com 
be higher [2-5]. The outcome of simple decompressive operations are poor or insufficient [6-8]. Especially after laminectomy, the fibrous tissue on the spinal dura leads to band formation and, by tightly surrounding the dura, may lead to discomfort or pain in the back and sometimes neurological injury $[9,10]$. Although autograft use has advantages for fusion, it causes donor site morbidity $[11,12]$. Preliminary 3-year follow-up results of the three-column fusion technique (Lumbar laminoplasty technique) convenient with the current minimal invasive surgery concept were presented 3 years ago [13]. Here, we present the long-term results (5.5 years on average).

\section{Materials and Methods}

Forty patients with one level grade 1 and 2 ISL operated on between February 2003 and December 2009 at ourclinic were included. All patients were evaluated clinically and radiologically. All patients underwent preoperative and postoperative X-ray, computed tomography (CT) and magnetic resonance imaging (MRI). All patients were conservatively treated for 6 months preoperatively by bed rest, medical treatment, physical therapy and/or lumbosacral corset use. Surgery was done for patients that did not respond to conservative treatment. Visual analog scale (VAS), and the Prolo economical and functional scale was used for follow-up [14]. All patients had upright dynamic $\mathrm{X}$-rays taken in which sagittal plane rotation and slipping, and disc space heights were measured. The finalaverage follow-up time was 5.5 years. Preoperative and postoperative standard anteroposterior, lateral, oblique and upright dynamic X-rays were routinely taken. The surgical technique was the same as described in a preliminary report and was not modified.

\section{Clinical and radiological follow-up}

All patients were clinically and radiologically evaluated at the postoperative day 1, 6 months, 1, 3, and 5 years. VAS, and the Prolo economical and functional scales used for clinical follow-up. Upright dynamic X-rays and lumbar vertebral CT with sagittal and coronal reconstruction were used for radiological follow-up. The quality of fusion was determined on the coronal reconstruction of lumbar vertebral CT and all paients were followed-up until fusion was present. Additionally, to detect any adjacent segment disease, non-contrast lumbar MRI was performed in the long-term follow-up.

\section{Results}

Twenty one (52.5\%) female and 19 (47.5\%) male patients were included in the study. Mean age was 43.5 years (range, 22-57 years). The most common symptoms were back pain (89\%), pelvic and leg pain (69\%) and reduction in the walking distance (65\%). A total of 180 pedicle screws were inserted in 40 patients; posterior lumbar interbody fusion (PLIF) and laminoplasty with reduction was performed in 20 patients for L4-L5, in 12 patients for L5-S1, in 4 patients for L3-L4-L5 and in 4 patients for L4-L5-S1. Ten (25\%) patients with ISL had accompanying spinal stenosis. The difference between preoperative and postoperative sagittal plane dislocation and disc space height values were statistically significant in all patients $(p<0.05)$. Solid grade 4 fusion was observed in 38 patients; grade 2 pseudoarthrosis developed in $2(5 \%)$ patients, but both were asymptomatic. The average duration of follow-up was 5.5 years.

None of the patients had any motor deficit. Straight leg raising test was positive in 11 patients (27.5\%). Hyporeflexia of the achille was detected in 6 (15\%) patients.

Preoperative clinical, economical and radiological findings are summarized in Table 1. In two cases, the anteriorly placed allograft worn out or pseudoarthrosis was present. These patients had no pain to support pseudoarthrosis clinically; fusion was radiologically insufficient but clinical success was achieved. Fusion was complete in all

Table 1. Postoperative rates of Prolo scores

\begin{tabular}{|c|c|c|c|c|c|c|}
\hline Evaluation & Preoperative & Postoperative & $3 \mathrm{mo}$ & $1 \mathrm{yr}$ & $5 \mathrm{yr}$ & Difference (\%) \\
\hline Motor deficit & None & None & None & None & None & 0 \\
\hline Sensory deficit & 10 & 5 & 3 & 3 & 3 & 36 \\
\hline Economical score & 2.5 & 3.6 & 4.1 & 3.8 & 3.8 & 67 \\
\hline Functional score & 3.1 & 3.4 & 3.2 & 3.2 & 2.5 & 64 \\
\hline
\end{tabular}


Table 2. Lumbar vertebra sagittal slipping and disc height values of cases

\begin{tabular}{lccc} 
Evaluation & Preoperative & Postoperative & $p$-value \\
Sagittal-plane slipping $(\%)$ & $30.46 \pm 7.2$ & $3.4 \pm 1.1$ & $<0.05$ \\
\hline Disc space height $(\%)$ & $19.01 \pm 2.1$ & $6.0 \pm 3.1$ & $<0.05$ \\
\hline
\end{tabular}

Table 3. Preoperative and postoperative VAS values

\begin{tabular}{lcccccc} 
Evaluation & Preoperative & Postoperative & $3 \mathrm{mo}$ & $1 \mathrm{yr}$ & $5 \mathrm{yr}$ & $p$-value \\
\hline Leg & 7.4 & 1.1 & 0.8 & 0.6 & 1.1 & $<0.05$ \\
\hline Back & 6.2 & 5.2 & 1.2 & 1.3 & 1.5 & $<0.05$ \\
\hline
\end{tabular}

VAS, visual analog scale.

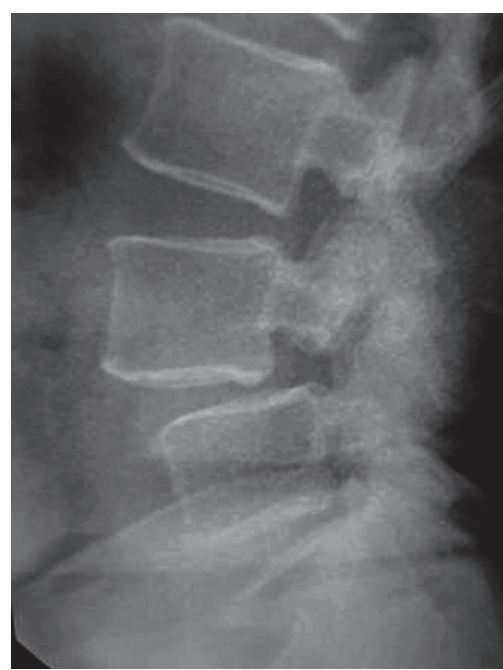

Fig. 1. Lateral X-ray shows the lysthetic segment at L4-L5 level.

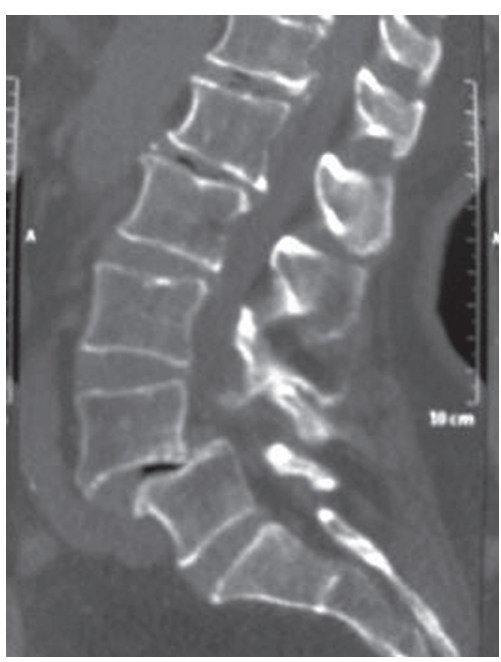

Fig. 2. Lateral (sagittal) lumbar vertebral computed tomography scan reveals the lysthetic segment at L4-L5 level. the other patients. Four patients were long-time smokers and smoked a pack of cigarettes per day. The fusion rate was $95 \%$ under these conditions.

Preoperative and postoperative values of sagittal slipping and disc space heights are shown in Table 2. VAS values are shown in Table 3. The differences between preoperative and postoperative sagittal plane dislocation and disc space height values were statistically significant $(p<0.05$, paired samples $t$-test).

Laceration of the dura was found in two cases (5\%); both cases were repaired with direct sutures. There was no problem in long-term follow-up. There was no infection. The mean operation time was 2 hours in the beginning and decreased to 65 minutes with experience. Mean amount of blood loss was 1.6 units. In long-term followup, no adjacent segment degeneration was observed for 3 years. Adjacent segment degeneration was detected above the level of fusion in $4(10 \%)$ patients at year 4 , in $6(15 \%)$ patients at year 5 and below the level of fusion in 3 (7.5\%) patients at year 5. Among all cases, the risk of adjacent segment degeneration was $32.5 \%$; 2 patients were accepted to have adjacent segment disease and were followed-up and medically treated.

\section{Illustrative case}

A 44 year-old female patient with a 4-year history of back pain that had recently increased in severity was diagnosed as Grade 2 ISL (L4-L5) by X-ray examination (Fig. 1). The patient's VAS score was $7 / 10$ for the back and Prolo score was $45 \%$. Lumbar vertebral CT revealed a Grade 2 bilateral lytic listhesis (Fig. 2). During the operation, the 


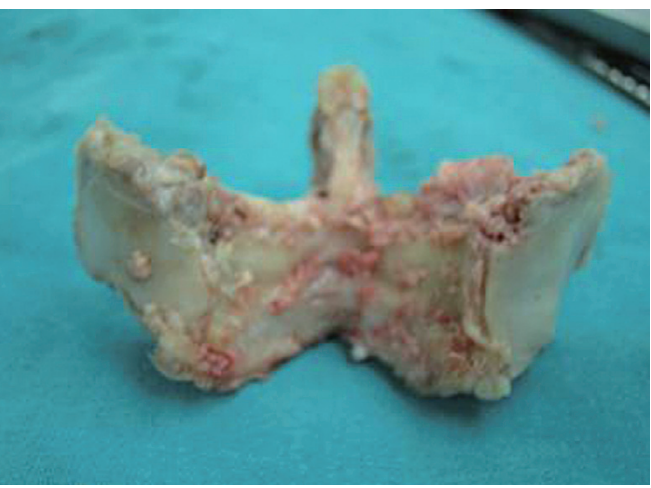

Fig. 3. The laminae after en-bloc resection.

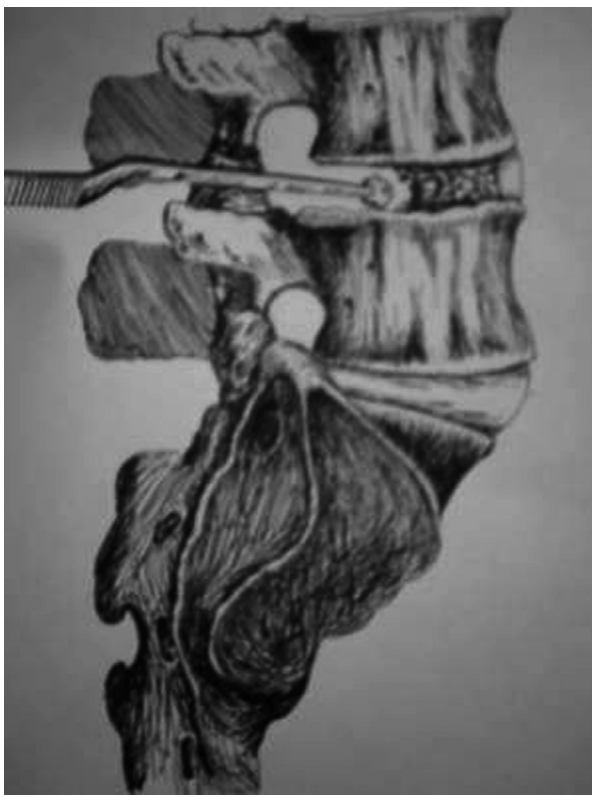

Fig. 4. Allograft insertion to the intervertebral space.

laminae were removed en-bloc (Fig. 3). After aggressive discectomy, allograft bone particles were inserted in the intervertebral disc space (Fig. 4). The pedicle screws were placed and tightened at the compressive phase and laminae were reinserted between the rods. The operation was ended after the placement of a drain. The early X-ray and sagittal lumbar vertebral CT scan revealed complete reduction (Fig. 5). Early first year and fifth year axial lumbar vertebral CT scans reveal the position of the laminae (Figs. $6,7)$. The VAS score was 1 and Prolo score was $15 \%$.

\section{Discussion}

Treatment modalities in ISL arediverse. The efficacy of the different surgical techniques remains contentious. The

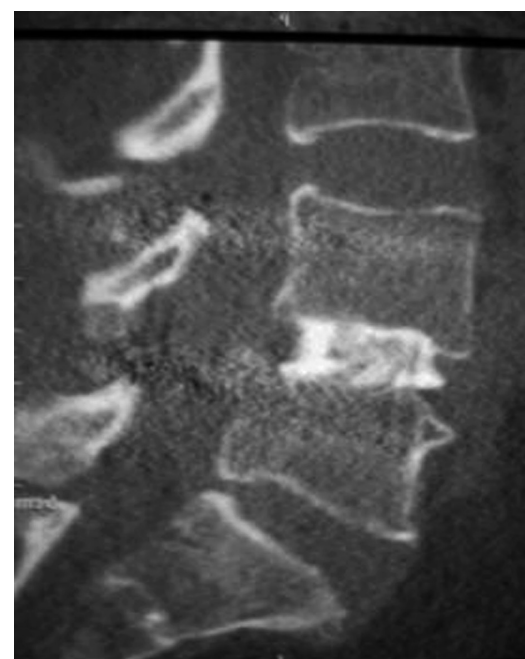

Fig. 5. Lateral (sagittal) lumbar vertebral computed tomography scan at 6 months postoperatively. Achievement of the restorative sagittal balance after the insertion of the allograft and compression of the level.

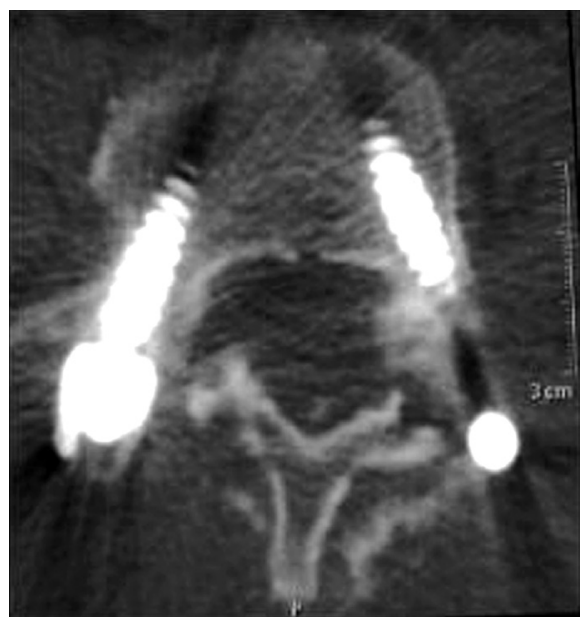

Fig. 6. The laminae 3 months after replacement viewed in an axial lumbar vertebral computed tomography scan.

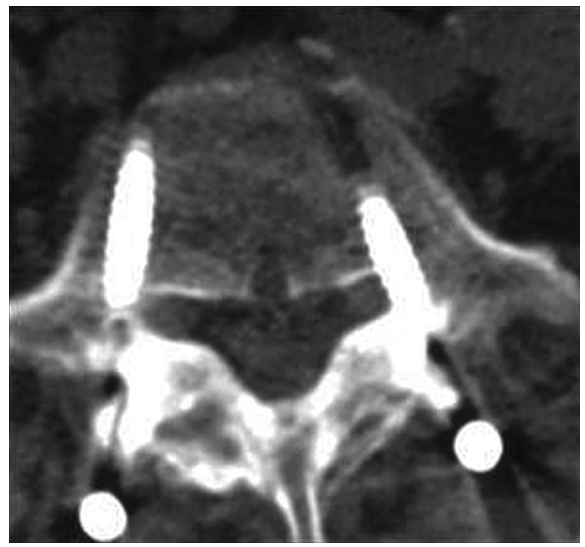

Fig. 7. Fusion of the laminae is visible at 5 years in an axial lumbar vertebral computed tomography scan. 
described techniques include posterior or posterolateral fusion follow simple decompression and reduction [15], PLIF with fixation with reduction [16], 360 degree circumferential fusion with reduction [17], simple decompression without fusion [18], posterior reduction and stabilization with minimal laminectomy [19] and reduction and stabilization without laminectomy [2,17]. A prospective randomized study reported a worse outcome for patients who underwent laminectomy and lost the support of the posterior column, compared to patients treated by arthrodesis [17]. In a comparative study, in low grade ISL, the outcome was better after anterior fusion together with posterior fusion than only with posterior fusion [20].

The PLIF technique was first descibed in 1946 as an effective and safe means of correcting sagittal balance; however, others have suggested a higher risk of dural tear and nerve injury [5]. Furthermore, compared to posterolateral fusion, PLIF was reported to have a better outcome [3]. Although nerve injury risk is reported to be higher with this technique, no such complication was evident in our series. Different intervertebral implants have been used for PLIF and the resulting fusion rates were $72 \%$ and $87 \%$ $[6,21]$. There is no comparative series about the allografts or autografts filled in these implants. The most important advantage of achieving a compact support with allograft bone particles is the enhancement of disc space height by the grafts and the protective effect against screw load. Autografts are the golden standard for osteosynthesis, with a high success rate for fusion. The rate of fusion is reported to be the same $(75 \%-95 \%)$ with autograft or allograft in ISL [15-27]. In our series, the fusion rate was higher than reported elsewhere. As radiologically poor fusion was observed in only two cases, the fusion rate was $95 \%$.

The successful fusion rate after osteosynthesis with autograft necessitates a long and difficult operation with more blood loss $[6,26]$. But, with lumbar laminoplasty, donor site morbidity is not a concern as allograft bone particles are used. This is very important for early mobilization. In this technique, the continuity of the physiological vertebral column was achieved posteriorly with laminoplasty procedure providing fusion and with pedicle screws the strength of the vertebral column is restored. This procedureis done without the need of anterior approach or cage insertion, eliminating complications like dural tear or nerve injury. This technique is carried out similar to the PLIF technique and adequate foraminotomy and spinal canal decompression are possible, so no disad- vantage exists.

The present study offers a long-term prospective of lumbar laminoplasty technique involving PLIF with allograft bone particles, protecting the laminae and interspinous ligaments that provide the integrity of the posterior column. A previous preliminary report of 20 cases described the short-term outcome [26]. The objective of the technique is massive discectomy after dissection of the facet joint as well as the lamina (en-bloc dissection of the lamina).

One of the many advantages of this technique is preventing psudocallus or callus formation at the fractured ends that interfere with fusion by their resection during the dissection and replacement of the laminae. A benefit of the resection of lamina is that the pars fracture zone with synovial tissue looks better before. Thus, the pars fracture line was opened with laminoplasty to permit better pars repair. Better fusion could be anticipated from a pars fracture.

No implant is needed to replace the laminae; they are placed between the rod and screws and are sutured to the interspinous ligaments. In addition, no implant, such as a cage, is needed forthe intervertebral disc space. This should reduce the expense of the surgery compared to other fusion techniques. The success rate of the technique defined as reposition of the protected posterior elements in lumbar degenerative spondylolisthesis is high and the procedure is easy [4].

Autograft versus allograft usefor successful fusion is still controversial $[11,25,26]$. But, lack of donor site complication, shorter duration of operation, less blood loss as well as the same rates of fusion have been described $[19,25,26]$. Thus, in our opinion, it is convenient to the current modern minimal invasive spinal surgery concept.

\section{Conclusions}

ISL lumbar laminoplasty technique has the advantages of shorter duration of operation, lack of graft donor site complications, protection of posterior column osseoligamentous structures, achievement of high fusion rates in one session with posterior approach and enhancement of tha sagittal balance and disc space height, with only one disadvantage of adjacent segment degeneration observed in one-third of the cases in the present long-term followup. The finding that symptomatic degeneration occurred in only $5 \%$ of case is a positive feature. In our opinion, this 
surgical technique is an effective and safe procedure.

\section{Conflict of Interest}

No potential conflict of interest relevant to this article was reported.

\section{Acknowledgments}

I contributed to conception and design, manuscript preparation, read and approved the final manuscript. The authors are thankful to Dr. Yıldıray Savaş (RadiologistIstanbul) for his helpful advice and for helping with radiologic evaluation of this study.

\section{References}

1. Moller H, Hedlund R. Instrumented and noninstrumented posterolateral fusion in adult spondylolisthesis--a prospective randomized study: part 2. Spine (Phila Pa 1976) 2000;25:1716-21.

2. Benini A, Plotz G. Reduction and stabilization without laminectomy for unstable degenerative spondylolisthesis: a preliminary report. Neurosurgery 1995;37: 843-4.

3. Fraser RD. Interbody, posterior, and combined lumbar fusions. Spine (Phila Pa 1976) 1995;20:167S177 S.

4. Matsudaira K, Yamazaki T, Seichi A, et al. Spinal stenosis in grade I degenerative lumbar spondylolisthesis: a comparative study of outcomes following laminoplasty and laminectomy with instrumented spinal fusion. J Orthop Sci 2005;10:270-6.

5. Sears W. Posterior lumbar interbody fusion for lytic spondylolisthesis: restoration of sagittal balance using insert-and-rotate interbody spacers. Spine J 2005;5: 161-9.

6. Csecsei GI, Klekner AP, Dobai J, Lajgut A, Sikula J. Posterior interbody fusion using laminectomy bone and transpedicular screw fixation in the treatment of lumbar spondylolisthesis. Surg Neurol 2000;53:2-6.

7. Ka-Siong Kho V, Chen WC. Posterolateral fusion using laminectomy bone chips in the treatment of lumbar spondylolisthesis. Int Orthop 2008;32:115-9.

8. Kho VK, Chen WC. The results of posterolateral lumbar fusion with bone chips from laminectomy in patients with lumbar spondylolisthesis. J Chin Med
Assoc 2004;67:575-8.

9. Lee TC. Reduction and stabilization without laminectomy for unstable degenerative spondylolisthesis: a preliminary report. Neurosurgery 1994;35:1072-6.

10. Wiens R, Rak M, Cox N, et al. Synchrotron FTIR microspectroscopic analysis of the effects of antiinflammatory therapeutics on wound healing in laminectomized rats. Anal Bioanal Chem 2007;387:167989.

11. Aghi MK, Walcott BP, Nahed BV, et al. Determinants of initial bone graft volume loss in posterolateral lumbar fusion. J Clin Neurosci 2011;18:1193-6.

12. Younger EM, Chapman MW. Morbidity at bone graft donor sites. J Orthop Trauma 1989;3:192-5.

13. Kotil K, Akcetin M, Tari R, Ton T, Bilge T. Replacement of vertebral lamina (laminoplasty) in surgery for lumbar isthmic spondylolisthesis: a prospective clinical study. Turk Neurosurg 2009;19:113-20.

14. Prolo DJ, Oklund SA, Butcher M. Toward uniformity in evaluating results of lumbar spine operations: a paradigm applied to posterior lumbar interbody fusions. Spine (Phila Pa 1976) 1986;11:601-6.

15. Booth KC, Bridwell KH, Eisenberg BA, Baldus CR, Lenke LG. Minimum 5-year results of degenerative spondylolisthesis treated with decompression and instrumented posterior fusion. Spine (Phila Pa 1976) 1999;24:1721-7.

16. Ali Y, Najmus S, Rahman M, Mhamud S. Short-term outcome of transforaminal lumbar interbody fusion for lytic and degenerative spondylolisthesis. J Orthop Surg (Hong Kong) 2012;20:371-4.

17. Thomsen K, Christensen FB, Eiskjaer SP, Hansen ES, Fruensgaard S, Bunger CE. 1997 Volvo Award winner in clinical studies: the effect of pedicle screw instrumentation on functional outcome and fusion rates in posterolateral lumbar spinal fusion: a prospective, randomized clinical study. Spine (Phila $\mathrm{Pa}$ 1976) 1997;22:2813-22.

18. Herron LD, Trippi AC. L4-5 degenerative spondylolisthesis. The results of treatment by decompressive laminectomy without fusion. Spine (Phila Pa 1976) 1989;14:534-8.

19. Bednar DA. Surgical management of lumbar degenerative spinal stenosis with spondylolisthesis via posterior reduction with minimal laminectomy. J Spinal Disord Tech 2002;15:105-9.

20. Swan J, Hurwitz E, Malek F, et al. Surgical treatment 
for unstable low-grade isthmic spondylolisthesis in adults: a prospective controlled study of posterior instrumented fusion compared with combined anterior-posterior fusion. Spine J 2006;6:606-14.

21. Miyakoshi N, Abe E, Shimada Y, Okuyama K, Suzuki T, Sato K. Outcome of one-level posterior lumbar interbody fusion for spondylolisthesis and postoperative intervertebral disc degeneration adjacent to the fusion. Spine (Phila Pa 1976) 2000;25:1837-42.

22. Harvey CJ, Richenberg JL, Saifuddin A, Wolman RL. The radiological investigation of lumbar spondylolysis. Clin Radiol 1998;53:723-8.

23. Kim NH, Lee JW. Anterior interbody fusion versus posterolateral fusion with transpedicular fixation for isthmic spondylolisthesis in adults. A comparison of clinical results. Spine (Phila Pa 1976) 1999;24:812-6.

24. Nugent PJ, Dawson EG. Intertransverse process lum- bar arthrodesis with allogeneic fresh-frozen bone graft. Clin Orthop Relat Res 1993;(287):107-11.

25. Gibson S, McLeod I, Wardlaw D, Urbaniak S. Allograft versus autograft in instrumented posterolateral lumbar spinal fusion: a randomized control trial. Spine (Phila Pa 1976) 2002;27:1599-603.

26. Haid RW Jr, Branch CL Jr, Alexander JT, Burkus JK. Posterior lumbar interbody fusion using recombinant human bone morphogenetic protein type 2 with cylindrical interbody cages. Spine J 2004;4:527-38.

27. Omidi-Kashani F, Hasankhani EG, Rahimi MD, Khanzadeh R. Comparison of functional outcomes following surgical decompression and posterolateral instrumented fusion in single level low grade lumbar degenerative versus isthmic spondylolisthesis. Clin Orthop Surg 2014;6:185-9. 